\title{
Medical Image of the Month: Mounier-Kuhn Syndrome
}

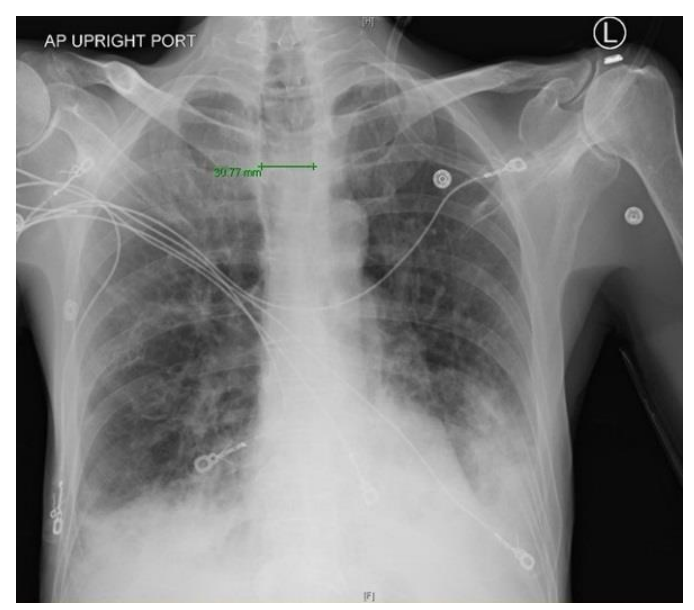

Figure 1. AP chest x-ray showing significant tracheomegaly (diameter $30.8 \mathrm{~mm}$ ), bilateral interstitial infiltrates with dense consolidation more at the lower lobes (left>right).

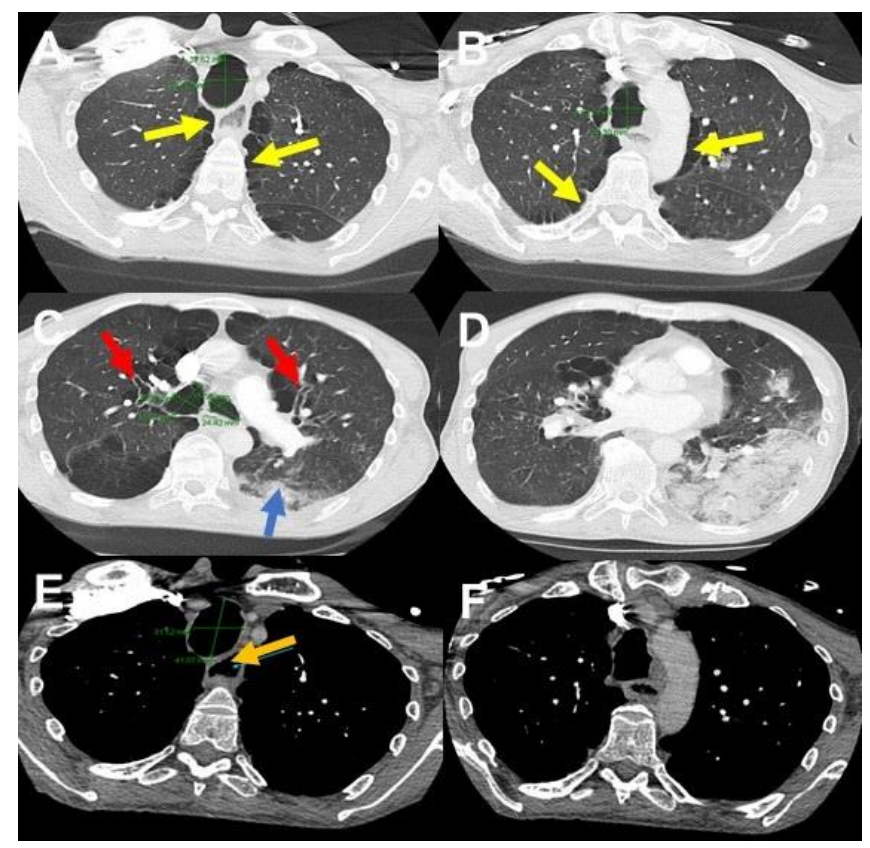

Figure 1. Axial thoracic $C T$ in lung windows $(A-D)$ and soft tissue windows $(E-F)$. Sagittal CT in soft tissue windows $(\mathrm{G}-\mathrm{H})$. A: tracheal diameters in 2 dimensions (coronal $30.4 \mathrm{~mm}$, sagittal $37.6 \mathrm{~mm}$ ), para-septal emphysema (yellow arrows). B: showing tracheomegaly $(23.2 \times 34.3 \mathrm{~mm})$ and para-septal emphysema changes (yellow arrows. C: enlarged mainstem bronchi diameters (right mainstem $22.3 \times 30.6 \mathrm{~mm}$, left mainstem $24.4 \times 16.0 \mathrm{~mm}$ ). In addition to central bronchiectatic changes (red arrows), left lower lobe consolidative changes (blue arrow). D: dense left lower lobe consolidation and para-septal emphysema. E: Significant tracheomegaly $(31.5 \times 41 . \mathrm{mm})$ and dilated esophagus (orange arrow). F: Significant tracheomegaly and dilated esophagus. 


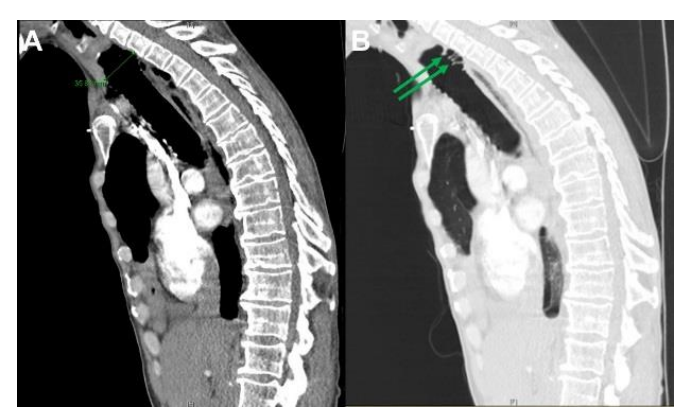

Figure 3. A: Sagittal CT scan (soft tissue window) showing significant tracheomegaly (sagittal diameter $35.8 \mathrm{~mm}$ ). B: Sagittal CT chest (lung window) showing significant tracheomegaly, multiple tracheal diverticuli (green arrows) on the upper posterior tracheal wall.

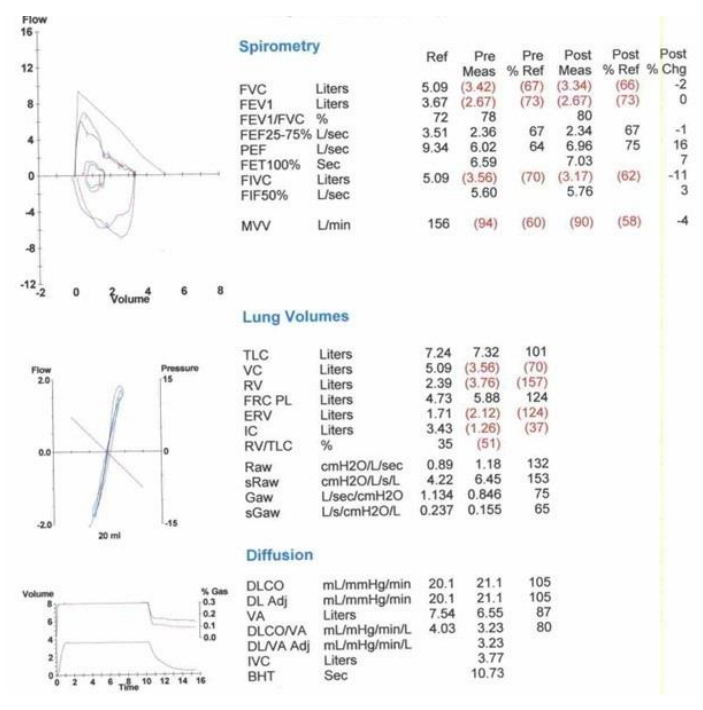

Figure 4. Pulmonary function testing.

A 52-year-old non-smoking, Caucasian male patient with a past medical history of reported chronic obstructive pulmonary disease (COPD), recurrent lower respiratory tract infections, prior history of pneumothorax, and dysphagia presented with fevers and shortness of breathing associated with a productive cough for one week. Clinically, he was mildly tachypneic and chest auscultation revealed crackles bilaterally - more prominent at the left base. A chest radiograph (Figure 1) showed bilateral lower lobe pulmonary opacities (left more than right). Computed tomography (CT) of the chest demonstrated airspace disease in the lower lobes in addition to significant tracheobronchomegaly along with paraseptal emphysema and central bronchiectatic changes (Figures 2 and 3). Upper posterior tracheal wall diverticulae were also noted (Figure 3). Serum $\alpha_{1}$-antitrypsin level and serum immunoglobulins, including lgE levels, were normal. Our patient declined performing diagnostic bronchoscopy. He had a pulmonary function test performed few months prior to his hospital admission which showed combined mild obstructive/restrictive pattern (Figure 4). He responded well to empiric antibiotics and chest percussion therapy. He was discharged in stable condition. 


\section{Discussion}

On the basis of above findings, a diagnosis of Mounier-Kuhn syndrome complicated by pneumonia was made. The syndrome was first described by P. Mounier-Kuhn in 1932

(1). The diagnosis is usually made when the tracheal diameter is greater than $3 \mathrm{~cm}$ on a CT chest (measured $2 \mathrm{~cm}$ above the aortic arch) (2). Other diagnostic criteria include a mainstem bronchial diameter of 20-24 mm (right) and 15-23 mm (left) (3). Our patient's tracheal diameter was around $37 \mathrm{~mm}$. Both mainstem bronchi were dilated.

The abnormal tracheobronchial dilatation in this syndrome is attributed to atrophy of the muscular and elastic tissues in the tracheal and the bronchial walls (3). Hence, in addition to tracheobronchomegaly, these patients can also develop tracheal diverticulosis along with varicose and cystic bronchiectasis (3). These patients usually present in the 3rd or 4th decade of life with nonspecific respiratory symptoms including recurrent bronchitis and subsequently end up being misdiagnosed with COPD (3).

Three subtypes of this syndrome had been described. Subtype 1 has symmetric dilation of the trachea and mainstem bronchi. Subtype 2 demonstrates tracheal dilation and tracheal diverticula. Subtype 3 has diverticular and saccular structures extending to the level of the distal bronchi (3). Our patient likely fits subtype 3 of this syndrome. Overall, treatment is supportive - usually with antibiotics, physiotherapy and postural drainage. In rare instances, tracheal stenting has been used (4). Special consideration should be taken post intubation as achieving good cuff seal can be potentially challenging.

Dysphagia has not been well documented in this syndrome and could be a coincidental finding in our case. However, theoretically, the etiology of this patient's dysphagia could be secondary to extrinsic compression of the anterior esophageal wall by his markedly dilated trachea. Historically, he underwent multiple esophageal dilatations and at least one Botox injection over the last 5 years without any significant improvement.

\section{Abdulmonam Ali MD and Naga S. Sirikonda MD}

Pulmonary and Critical Care

Good Samaritan Hospital

Mount Vernon, Illinois

\section{References}

1. Mounier-Kuhn P. "Dilatation de la trachee: constatations, radiographiques et bronchoscopies." Lyon Med. 1932;150:106-9.

2. Menon B, Aggarwal B, lqbal A. Mounier-Kuhn syndrome: report of 8 cases of tracheobronchomegaly with associated complications. South Med J. 2008;101(1):837. [CrossRef] [PubMed]

3. Falconer M, Collins DR, Feeney J, Torreggiani WC. Mounier-Kuhn syndrome in an older patient. Age Ageing. 2008;37(1):115-6. [CrossRef] [PubMed]

4. Schwartz M, Rossoff L. Tracheobronchomegaly. Chest 1994;106(5):1589-90. [CrossRef] [PubMed] 